\title{
Demographic Transition, Health Care Challenges, and the Impact of Emerging International Regulatory Trends With Relevance to Regenerative Medicine
}

\author{
Gil Van Bokkelen ${ }^{1}$ • Manal Morsy ${ }^{1}$ - Toshi-hiko Kobayashi ${ }^{1,2}$
}

Published online: 23 May 2015

(C) Springer International Publishing AG 2015

\begin{abstract}
Unprecedented demographic trends threaten to have a major impact on the practice of medicine and the application of limited health care resources in the years ahead. As the elderly segment of the population greatly expands globally, the incidence and prevalence of various agingrelated diseases and conditions are also expected to substantially increase, while health care resources become progressively strained. This threatens to have an adverse impact on national health care systems and patient quality of life. Regenerative medicine technology has the potential to provide an effective solution for some of these challenges, by enabling new and more effective ways to treat costly and debilitating conditions, particularly in the cardiovascular and neurological areas. Emerging international regulatory trends, some specifically focused on regenerative medicine, could help enable a wave of innovation that could promote the development of more effective solutions that improve clinical care, enhance patient quality of life, and reduce overall health care costs.
\end{abstract}

Keywords Regenerative medicine $\cdot$ Regulatory trends . Aging population $\cdot$ Health care innovation $\cdot$ Neurological disease $\cdot$ Cardiovascular disease

This article is part of the Topical Collection on Stem Cells: Policies from the Bench to the Clinic

Gil Van Bokkelen

gilvb@athersys.com

1 Athersys, Inc., 3201 Carnegie Ave, Cleveland, OH 44115-2634, USA

2 University of Tokyo, Pharmaceutical Sciences, Tokyo, Japan

\section{Introduction}

An aging global population threatens the viability of health care systems around the world. As a result of the aging of the population cohort born during the years 1945-1964 postWorld War II in combination with other trends, including the increase in life expectancy that occurred during the twentieth century and a long-term decline in birth rates, many developed nations are now experiencing an unprecedented demographic $\operatorname{shift}^{1}[1]$. This transition is driving a dramatic expansion of the elderly population in developed countries around the world that is expected to continue for the next several decades. ${ }^{2,3,4}$ Within the next several years, the number of people over age 65 will exceed the number of people under age 5 for the first time in recorded history.

According to Organisation for Economic Co-operation and Development (OECD) analysis, this expansion of the global elderly population will have a significant effect on health care systems, quality of life, and long-term economic prosperity. Demographic trends in Japan, the USA, and the European Union are particularly concerning. ${ }^{5}$ The segment of the population over the age of 65 is projected to double in size between the years 2010 and 2030, and national demographic profiles will be meaningfully altered. In some countries, such as Japan, the problem is rather daunting, as the population segment over the age of 65 already represents about $25 \%$ of the population, and

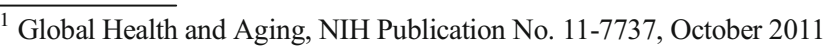
(National Institute on Aging)

${ }^{2}$ Health At A Glance 2013: OECD Indicators

${ }^{3}$ World Population Prospects-The 2012 Revision (United Nations, Department of Economic and Social Affairs)

${ }^{4}$ World Population Ageing 2013 (United Nations, Department of Economic and Social Affairs)

${ }^{5}$ China and India are also problematic, where the number of individuals over the age of 65 is expected to more than triple between the years 2010 and 2050 , to more than 550 million people.
} 
by 2030 , it will represent more than $30 \%$. The cohort of the population over the age of 80 will more than double, from $6 \%$ of the population in Japan to more than $14 \%$ within roughly two decades. In the USA, the group over the age of 65 is projected to grow by more than $80 \%$ between the years 2010 and 2030, representing an increase from 40 million individuals to more than 72 million people in only two decades. ${ }^{6}$ In Europe (i.e., the EU28), this segment of the population will swell to more than 125 million people by 2030 .

\section{Health Care Problems Posed by an Aging Global Population}

According to OECD analysis, this expansion of the global elderly population will have a significant effect on health care systems, quality of life, and long-term economic prosperity. ${ }^{8}$ Over time, as life expectancies have steadily increased, there has also been a shift in mortality and disability trends. As we get older, we become more susceptible to a range of agingrelated diseases and conditions, including cardiovascular disease, neurological conditions, certain chronic inflammatory and immune disorders, and others. ${ }^{9}$ Many age-associated diseases and conditions become progressively more debilitating over time. Consequently, the corresponding health care cost burden and impact on quality of life also increase with age. Evolving health care and social needs driven by these powerful demographic trends must ultimately be reflected in the systems and infrastructure for providing health care.

Health care economic analysis demonstrates that as we become more susceptible to aging-related diseases and conditions, the expense, health care infrastructure needs, and social burden escalate. According to data compiled by the National Center for Health Statistics, elderly cohorts in the USA spend four to ten times as much on annual health care-related expenses in comparison to young, healthy cohorts. ${ }^{10}$ This is a direct reflection of the fact that many aging-related diseases and conditions take a significant toll, in terms of adversely impacting quality of life, direct economic costs, and the escalating need for institutional support or home care for individuals that are partially or extensively disabled as a result of deteriorating health [2]. According to analysis conducted by the Alliance for Aging Research, $83 \%$ of health care spending is associated with chronic conditions. ${ }^{11}$

\footnotetext{
${ }^{6} 2010$ U.S. Census data and projections

${ }^{7}$ European Institute of Demography, European Demographic Data Sheet, 2010

${ }^{8}$ Why Population Aging Matters-A Global Perspective, NIH Publication No. 07-6134, March 2007 (National Institute on Aging)

${ }^{9}$ The Silver Book: Chronic Disease and Medical Innovation in an Aging

Population 2006, Alliance for Aging Research

${ }^{10}$ Health U.S.A., 2009

${ }^{11}$ The Silver Book: Chronic Disease and Medical Innovation 2009
}

While there have been many remarkable advances in medical care in recent decades, there are many disease indications that currently lack an effective form of treatment or intervention. For example, while there has been success in reducing or blunting the impact of certain forms of heart disease (e.g., with the rise of statins) and treating certain types of life-threatening acute events (e.g., improved infrastructure and techniques for treating patients that have suffered a myocardial infarction), advances in care that have meaningfully improved mortality in an acute setting have resulted in a shift toward patients suffering from chronic ischemia and a rise in heart failure [3]. In effect, this has shifted the burden from acute into chronic disease conditions that have a longer term impact and higher cost. The rise in obesity, both among adults and adolescents, and the corresponding increase in diabetes are also worrisome, given the correlation with cardiovascular disease and other related conditions that are leading causes of mortality and morbidity. The American Heart Association has projected that between 2010 and 2030, the direct costs of cardiovascular disease will triple, from $\$ 273$ billion to more than $\$ 818$ billion annually in the USA, and the total direct and indirect costs associated with cardiovascular disease will reach nearly $\$ 1.1$ trillion annually $[4 \bullet \cdot]$.

Perhaps more problematic is the steady increase in chronic neurological diseases and conditions. ${ }^{12}$ With the growth in the elderly segment of the population, the incidence, prevalence, and long-term impact of stroke, Alzheimer's disease, Parkinson's disease [5], and other causes of serious neurological disability are projected to meaningfully rise in the years ahead. Patients suffering from these conditions frequently require full-time institutional care or intensive supportive care at home, and in many cases, patients may require such support for many years, creating a long-term economic and quality of life "tail effect."

The projected economic burden associated with the demographic shift reflects the palliative nature of currently available forms of treatment for many of the most problematic conditions. While current forms of care provide some benefit to patients suffering from serious conditions, in many instances, these approaches fail to effectively address fundamental causes of the disease or provide durable relief. Therefore, such approaches inadequately impact the medical needs of the patient, resulting in the corresponding need for long-term supportive care that is resource intensive.

Ultimately, health care resources are inherently limited, both in terms of the available financial resources that may be allocated and skilled medical staff needed to provide the appropriate care. According to the Association of American Medical Colleges (AAMC), there is already a shortage in the number of primary care and specialty physicians in the USA, a

\footnotetext{
12 The Silver Book: Neurological Disease: Chronic Disease and Medical Innovation in an Aging Nation 2009 Alliance for Aging Research
} 
shortfall projected to grow to more than 91,500 physicians nationally by the year 2020 and more than 130,000 physicians by the year $2025 .{ }^{13}$ In addition, the US Government has projected a national shortage of over 1 million nurses by the year 2022. ${ }^{14}$ If nothing else changes, limited health care resources and increased demand will eventually result in mounting challenges in patient access to care and escalating costscreating an undesirable trend toward rationing of available resources.

A shortage of physicians and nurses is also occurring in Japan and the European Union. According to the Ministry of Health Labor and Welfare, hospitals in Japan require an additional 24,000 physicians $[6 \bullet, 7]$. In the EU, the European Commission projects there will be a shortage of 230,000 physicians and 590,000 nurses by the year 2020. This will result in an estimated $13.5-14 \%$ of needed care not being provided, simply because there are not enough caregivers to provide it $[8 \cdot]$.

\section{The Potential Impact of Innovative Regenerative Medicine Therapies}

Faced with the seemingly perfect storm of an aging population, rising demand for finite health care resources, and a growing shortage of physicians and nursing staff, a rather bleak picture emerges. However, recent regulatory trends and advances may be able to help overcome some of these difficulties, both by accelerating innovation and encouraging greater investment in innovative health care technology.

The efforts directed toward development of novel pharmaceuticals to treat established areas of substantial unmet medical need, especially those that will become more prevalent in an aging global society, seem to have achieved diminishing returns in recent years. This is reflected in part by increased cost of development, few approvals in the areas of greatest need, and lengthier development times. ${ }^{15}$

To date, no truly effective treatment exists for many cardiovascular conditions, including for patients who have experienced severe damage from myocardial infarction, and those that are at high risk for chronic ischemia, congestive heart failure, or chronic peripheral arterial or vascular disease. Similarly, despite the beneficial impact of some currently available treatments, highly effective and clinically practical treatments for many neurological conditions such as stroke, Alzheimer's disease, Parkinson's disease, and other conditions have remained elusive.

\footnotetext{
${ }^{13}$ AAMC Centers for Workforce Studies

${ }^{14}$ Bureau of Labor Statistics, Occupations with the largest projected number of job openings due to growth and replacement needs, 2012see http://www.bls.gov/news.release/ecopro.t08.htm

${ }^{15}$ Tufts Center for Drug Development-Innovation in the Pharmaceutical Industry: New Estimates of R\&D Costs
}

In recent years, there has been a shift from traditional pharmaceuticals toward biologics, as reflected by the rise in market share of monoclonal antibodies, which now represent many of the top-selling drugs [9]. ${ }^{16}$ Ultimately however, therapies that work through a single mode of action may be somewhat limited for more complex conditions that may require a form of therapy that can convey multimodal effects.

Regenerative medicine and cell therapy technologies are considered to have the potential to dramatically impact care in many areas of established unmet medical need, where current approaches are inadequate. ${ }^{17}$ Since cell therapies frequently act through multiple modes of action, it is believed these types of technologies may be able to overcome longstanding limitations of traditional "single-mode" therapies. There are a growing number of clinical trials evaluating regenerative medicine approaches for acute and chronic conditions in the cardiovascular, neurological, and other areas that are increasingly burdensome and relevant to an aging global population. These technologies have the potential to improve the standard of care, enhance clinical outcomes and patient quality of life, and improve the economic efficiency of health care systems as a whole. ${ }^{18}$ Given existing demographic trends and the escalating shortage of health care resources, as described above, the development of new forms of treatment that deliver meaningful improvements in clinical care and patient quality of life is a priority for many health care systems. Therapies that have the potential to change standard of care such that a better clinical outcome is possible, while also reducing the need for resource-intensive clinical intervention, nursing support, and institutional care, would allow finite resources to be used more effectively. If new regenerative medicine therapies can be developed in the next few years that help address major areas of unmet medical need, particularly in the neurological, cardiovascular, cancer therapy, renal disease, and inflammatory and immune areas, it could have a major effect on health care efficiency as a whole.

Recent regulatory initiatives suggest a growing recognition of the need to define and implement policies that have the potential to accelerate the development of safe and effective solutions to our most challenging health care needs [10]. Emerging regulatory programs in the USA, Japan, and the European Union reflect this trend and illustrate the growing appreciation for more efficient clinical development, while also maintaining the commitment to protecting patient safety (Table 1).

\footnotetext{
$\overline{16} \mathrm{http} / / /$ www.drugs.com/stats/top100/2013/sales

${ }^{17} 2014$ Regenerative Medicine Industry Report, from the Alliance for Regenerative Medicine

18 "The Application of Regenerative Medicine Products and Technologies Toward Areas of Significant Medical Need-Improving Outcomes and Reducing Costs"-Alliance for Regenerative Medicine, May 2013
} 
Table 1 Examples of regulatory initiatives designed to promote accelerated development and faster access for patients

\begin{tabular}{|c|c|c|c|}
\hline Pathway & Year implemented & Agency & Description \\
\hline $\begin{array}{l}\text { Conditional approval } \\
\text { for regenerative } \\
\text { medicine products }\end{array}$ & 2014 & PMDA & $\begin{array}{l}\text { Implemented to promote faster access to regenerative medicine therapies that } \\
\text { demonstrate safety and probable benefit - conditional approval possible following a } \\
\text { single clinical trial. Requires clinical evidence demonstrating safety and probable } \\
\text { therapeutic benefit. Enables reimbursement under national health care system }\end{array}$ \\
\hline $\begin{array}{l}\text { Adaptive licensing } \\
\text { pilot project }\end{array}$ & 2014 & EMA & $\begin{array}{l}\text { Exploratory program to promote access to new medicines for restricted patient } \\
\text { populations (i.e., niche indications). Not modality specific, but explicitly } \\
\text { contemplates cell and gene therapies. No clear linkage to reimbursement }\end{array}$ \\
\hline $\begin{array}{l}\text { Breakthrough Therapy } \\
\text { designation }\end{array}$ & 2012 & FDA & $\begin{array}{l}\text { Designed to promote faster access to new medicines for serious medical conditions } \\
\text { where standard of care is limited or inadequate or no effective therapy exists. } \\
\text { Requires preliminary clinical evidence for designation. Enables more frequent } \\
\text { interaction with FDA and potential for expedited clinical development }\end{array}$ \\
\hline $\begin{array}{l}\text { Conditional marketing } \\
\text { authorization }\end{array}$ & 2005 & EMA & $\begin{array}{l}\text { Enacted to promote accelerated development of therapies to seriously debilitating or } \\
\text { life-threatening diseases, emergency public health threats, and orphan indications. } \\
\text { Focused on areas of unmet need, requires positive benefit-risk assessment, and } \\
\text { benefit to providing immediate access outweighs the risks of need for additional data }\end{array}$ \\
\hline Fast track & 1997 & FDA & $\begin{array}{l}\text { Accelerated review of therapies designed to treat serious conditions. Designation } \\
\text { requires non-clinical or clinical data demonstrating the potential to treat an } \\
\text { established unmet medical need. Enables expedited clinical development, potential } \\
\text { for rolling regulatory submissions, and possibility of shortened review times }\end{array}$ \\
\hline $\begin{array}{l}\text { Approval under } \\
\text { exceptional } \\
\text { circumstances }\end{array}$ & 1993 & EMA & $\begin{array}{l}\text { Designed for medicines with urgent public health need. Requires "non-comprehensive" } \\
\text { evidence of safety and benefit. Enables shortened clinical review times, requires } \\
\text { annual benefit-risk assessment, and post-authorization data collection }\end{array}$ \\
\hline Priority review & 1992 & FDA & $\begin{array}{l}\text { Established for therapies intended to treat serious medical conditions. Requires } \\
\text { preclinical and/or clinical data. Enables shortened review time of } 6 \text { months } \\
\text { (from a 10-month standard) }\end{array}$ \\
\hline Accelerated approval & 1992 & FDA & $\begin{array}{l}\text { Designed for therapies to treat serious conditions. Enables use of surrogate end points } \\
\text { reasonably likely to predict a clinical benefit. Requires understanding of relationship } \\
\text { between drug effect and disease process. Primary benefit is shortened clinical } \\
\text { development, with post-marketing approval typically required }\end{array}$ \\
\hline Special medical use & $\begin{array}{l}\text { Under } \\
\text { consideration }\end{array}$ & FDA & $\begin{array}{l}\text { Envisioned as a form of adaptive licensing, this approach is under } \\
\text { consideration and could potentially be implemented initially for highly problematic } \\
\text { areas such as the development of new antibiotics for multidrug-resistant pathogens }\end{array}$ \\
\hline
\end{tabular}

\section{Regulatory Initiatives in the USA Designed to Accelerate Development of Safe and Effective Medicines}

In 1992, congress passed the Prescription Drug User Fee Act (PDUFA) into law, essentially as a 5-year pilot project. The intent of PDUFA was to help safely speed the development of innovative new medicines by providing additional resources to the FDA through user fees that would be paid by sponsors developing new medicines to support an increase in FDA staff. PDUFA has largely been hailed as a success, and in 2012, the legislation was renewed for the fourth time (PDUFA-V), with modifications designed to further promote more efficient development of innovative therapies that are safe and effective and that have the potential to improve the standard of care. ${ }^{19}$ In May of 2014, the FDA released its

\footnotetext{
${ }^{19}$ US Government Printing Office. Food and Drug Administration Safety and Innovation Act of 2012 (S-3187) <http://www.gpo.gov/fdsys/pkg/BILLS112s3187enr/pdf/BILLS-112s3187enr.pdf×. Accessed 28 August 2013.
}

"Guidance for Industry: Expedited Programs for Serious Conditions - Drugs and Biologics." This guidance conveys the key features of the expedited review programs including fast track designation, priority review, and accelerated approval as well as provides a through explanation of the new "Breakthrough Therapy" designation created by the Food and Drug Administration Safety and Innovation Act (FDASIA). ${ }^{20}$

FDASIA was enacted in July, 2012, to implement more broadly effective processes for the expedited development and review of innovative new medicines intended to address unmet medical needs. ${ }^{21}$ The newly established Breakthrough Therapy designation was enacted to address the need for alternative development pathways that help accelerate development of new treatments for serious or life-threatening unmet medical needs where standard of care is inadequate or an

\footnotetext{
${ }^{20}$ FDA Guidance (OMB Control Number 0910-0765) issued May, 2014, and available at http://www.fda.gov/downloads/drugs/ guidancecomplianceregulatoryinformation/guidances/ucm358301.pdf

${ }^{21} 21$ US Code $\S 356$-Expedited approval of drugs for serious or lifethreatening diseases or conditions
} 
effective therapy does not exist. The explicit intent is to accelerate the path to approval and avoid exposing patients for extended periods of time to less effective or active comparator drugs, and is meant to complement the other mechanisms including fast track, priority review, and accelerated approval. Breakthrough Therapy designation applies to investigational drugs that meet two criteria: (1) the drug is being studied for a serious, life-threatening disease or condition and (2) preliminary clinical evidence exists to support the designation, as demonstrated by preliminary clinical evidence that shows "...the drug may demonstrate substantial improvement over existing therapies on 1 or more clinically significant endpoints." Potential benefits include more frequent interaction with the FDA and the potential that a "...development program for the breakthrough therapy could be considerably shorter than for other drugs intended to treat the disease." Between July 2012 and March 2014, a total of 41 products, 40 by the Center for Drug Evaluation and Research (CDER) and 1 by the Center for Biologics Evaluation and Research (CBER), received designations out of a total of 133 applications to CDER and 22 to CBER [11].

In addition, in a continued effort to further broaden the accelerated approval framework, a proposed pathway under consideration by the FDA is an expedited development path that would lead to early approval predicated on restricted use, referred to as a "Special Medical Use" or SMU designation. This approach, which has not yet been implemented, may initially be focused on the development of therapies for highly problematic areas, such as the development of antibiotic therapies for multidrug-resistant pathogens, referred to as limited population antibacterial drug (LPAD). The concept is to allow development in a very narrow aspect of a disease, for example multidrug-resistance TB where a limited clinical program is conducted supported by in vitro and in vivo modeling, data from study(ies) in drug-sensitive infections leading to a special approval. ${ }^{22}$

All such approaches are geared to facilitate effective and frequent interactions with the FDA with the purposeful goal of expediting the drug development path and making effective, safe drugs accessible to patients much sooner than might be possible using the traditional development path.

\section{The Emphasis on Regenerative Medicine in Japan}

Long-term demographic trends are particularly challenging in Japan, where the expansion of the elderly population cohort is projected to be pronounced over the next several decades.

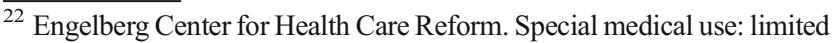
use for drugs developed in an expedited manner to meet unmet medical need. $<$ http://www.brookings.edu/search? start=1\&q=special + medical + use $>$ (2013).
}

This represents a formidable challenge to the national health care system in Japan that potentially threatens the long-term financial solvency of the system.

After evaluating potential ways to approach the problem, in 2013, the government and policy leadership announced an important new initiative that was founded on the assessment that regenerative medicine technology is viewed as offering greater potential to address areas of serious unmet medical need that any other area of technology evaluated $[12] .{ }^{23} \mathrm{Un}$ fortunately, it has also been recognized that in the past decade, few companies active in the area of regenerative medicine have been conducting clinical trials in Japan.

To help address this, the Ministry of Health Labor and Welfare (MHLW) and the Pharmaceuticals and Medical Devices Agency (PMDA) announced the intention to implement a novel and visionary regulatory framework designed to enable accelerated conditional approval of regenerative medicine therapies (Fig. 1). Traditional clinical development customarily requires phase I evaluation of safety, phase II trials to further evaluate safety and provide an initial assessment of efficacy, and typically at least two phase III larger scale trials to confirm safety and efficacy, a process that frequently requires years of development and substantial financial resources. Under the new framework, qualified regenerative medicine therapies could receive accelerated conditional approval after a single clinical study, provided the trial demonstrates the therapy to be safe and there is also evidence of a probable therapeutic benefit. Importantly, the new regulatory framework was explicitly designed to speed development of innovative regenerative medicines, as well as accelerate patient access by speeding commercialization of qualified therapies $[13,14 \bullet \cdot]$.

In November of 2013, the new legislation authorizing the establishment of the new framework was approved with overwhelming support by both houses of the Japanese Diet and was subsequently passed into law by the Japanese government, and in November 2014, the new framework went into effect $^{24}[15,16]$. Importantly, new therapies approved under the framework are also eligible for reimbursement under the national health care system in Japan.

The potential impact of the new framework in Japan on the field of regenerative medicine could be profound. The approach is designed to dramatically accelerate patient access to new medicines, while also making it possible for sponsors to meaningfully shorten clinical development times and reduce development costs. This initiative should also promote

\footnotetext{
${ }^{23}$ Japan policy of pharmaceutical innovation and regulatory science. Presentation during Pharmaceutical \& Regulatory Sciences Workshop at Harvard University School of Public Health, by Toshio Miyata, M.D., from MHLW, May 23rd-24th, 2013.

${ }^{24}$ See "Act to Revise Pharmaceutical Affairs Law (PAL) (Law No. 84/ 2013)" and the "Act Concerning Safety Assurance of Regenerative Medicine, etc." (Law No. 85/2013)
} 
Fig. 1 Novel approval system for regenerative medicine therapies in Japan. In 2013, the Japanese Diet passed two important pieces of legislation that could have a dramatic impact on the development of novel regenerative medicine therapies. The new framework makes it possible to obtain accelerated conditional approval of qualified regenerative medicines that are shown to be safe and where there is evidence of a probable therapeutic benefit. In November, 2014, the new system went into effect (from [16])

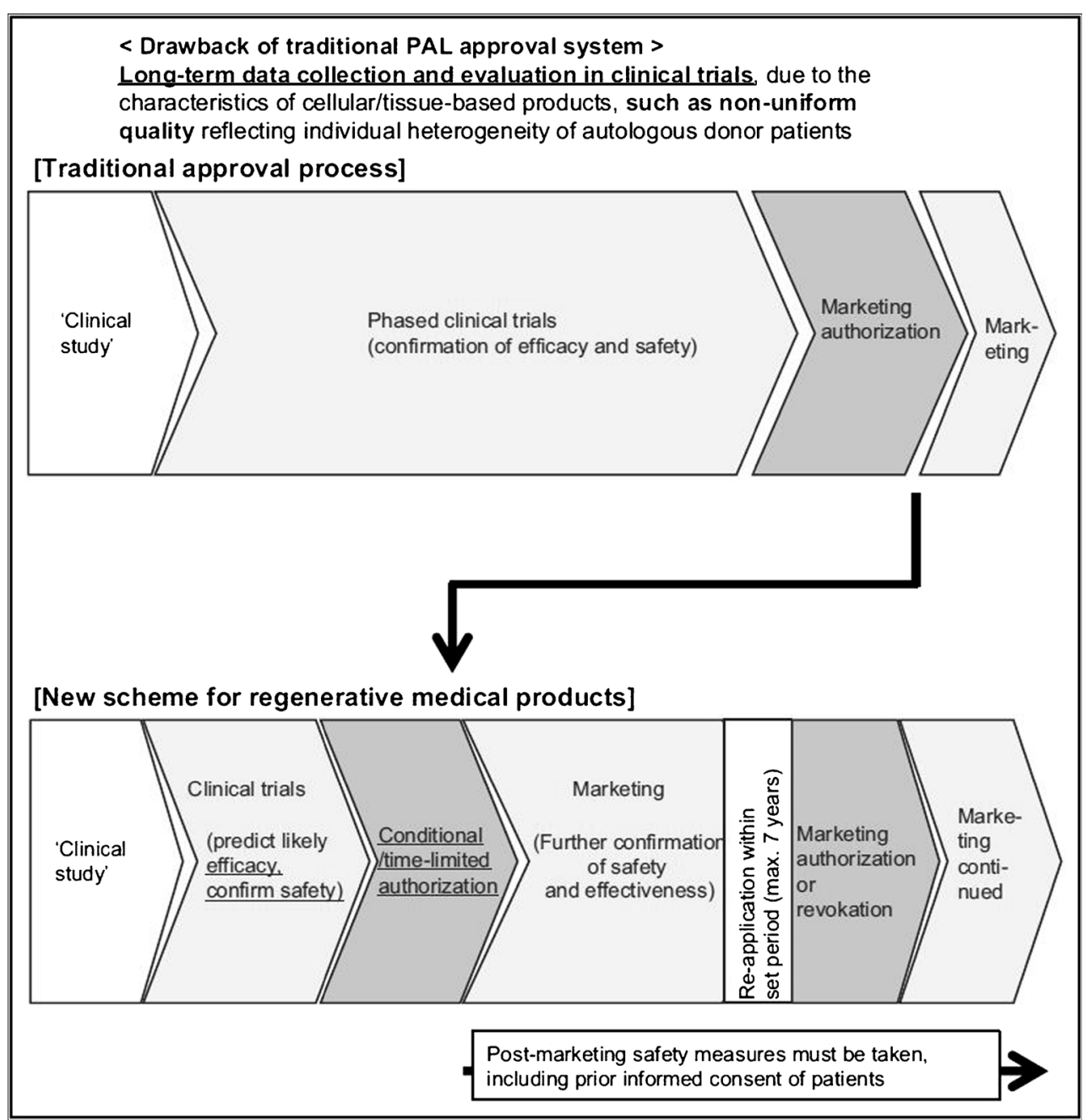

investment in the area, since faster and less expensive development and the ability to achieve accelerated commercialization would shift the risk-reward ratio in a favorable direction from an investment perspective. As a result, it could enhance patient access to regenerative medicine therapies and improve the efficiency and quality of care, while creating a powerful catalyst for development and promoting investment in Japan.

\section{The European Medicines Agency Pilot Program on Adaptive Licensing}

Recent developments in the European Union are also designed to promote accelerated development of novel therapies in established areas of serious unmet medical need. Conceptually similar to the initiative in Japan, the European Medicines Agency (EMA) has embraced the concept of an adaptive licensing initiative [17]. In March, 2014 the EMA announced a new pilot program, built on the concept of a "progressive licensing approach," also referred to as adaptive licensing $[18 \bullet \cdot$. The essential concept is to provide a form of contingent approval that speeds development and could also enable reimbursement but that also entails a strict commitment to conduct post-approval follow-up studies to gather additional data regarding safety and efficacy. Consistent with other regulatory initiatives designed to speed patient access to innovative new medicines while also ensuring patient safety, the clear emphasis is on facilitating advancement of "therapies that are being developed to address serious conditions where there is an unmet medical need, especially when there are no satisfactory alternative therapies." This new program is explicitly designed to "...maximize the positive impact of new drugs on public health...." EMA references a similar framework launched in the UK by the Medicines and Healthcare Products Regulatory Agency (MHRA), in March of 2014.

While the adaptive licensing program initiated by the EMA is meant to be broad and is not specifically designed to focus on or accommodate any specific type of therapy, EMA provided several illustrative case studies in the program announcement, to help demonstrate how the approach might work. One of these case studies illustrates how a genetically modified cell therapy designed to express growth factors that 
enhance healing and repair of cartilage, administered in combination with a matrix (i.e., representing a combined ATP), might be relevant for the approach.

The initiative recognizes that reimbursement represents a potential hurdle to implementation of the approach, and encourages the active involvement of third-party payers and health technology assessment bodies. Their endorsement will likely be a requirement for the approach to be successful. This is particularly true in areas where treatments may provide a substantial impact but also be expensive to implement.

\section{Conclusion}

Many countries around the world are now experiencing a significant expansion of the elderly segment of their populations. Since this group is susceptible to a range of agingrelated diseases and conditions that frequently require significant medical intervention and supportive care, it utilizes a greater proportion of health care resources than other population cohorts. The requirement for intensive clinical and supportive care is especially meaningful for acute and chronic conditions that may have a long-term impact on patient quality of life and require intensive long-term institutional supportive care, home care support, or both.

A seemingly inevitable consequence of the ongoing and unprecedented demographic shift is the need to deploy far greater health care resources toward the challenges posed by an expanding population of elderly. An unattractive alternative would be de facto rationing of the limited clinical and supportive care resources available, in conjunction with a greater emphasis on family-mediated home care and support.

Advances in clinical care and biopharmaceutical treatments have yielded dramatic successes in improving health care outcomes and patient quality of life in many indications. However, meaningful progress in many areas that threaten to become an escalating social, clinical, and economic burden has remained frustratingly elusive. Innovation in health care technology could yield safer and more effective treatments for areas of unmet medical need and offer the potential to greatly improve health care outcomes and enhance patient (and family) quality of life. New technologies, such as regenerative medicine, could also increase the efficiency of health care by reducing the need for clinical and long-term palliative institutional care, and alleviate the need for extended home care support. Rational pricing strategies and reimbursement policies will be critical aspects that influence the adoption of such therapies. Therapies that are administered only once, or perhaps a limited number of times, but that have the potential to deliver major improvements in clinical outcomes and substantially enhance patient quality of life may provide meaningful long-term benefits that may not be fully considered using traditional health care economic approaches. While such therapies may seem expensive relative to traditional pharmaceuticals, they may deliver a substantial benefit to cost ratio that fully justifies their adoption.

Regulatory initiatives adopted over the past several years in the USA, Japan, and the European Union have explicitly recognized the need for greater innovation focused on addressing established areas of serious unmet medical need. These new initiatives are designed to speed the development of safe and effective new treatments that could also help offset the impact of current demographic trends that pose a threat to social welfare and economic prosperity in many regions. New technologies such as cell therapy, gene therapy, and regenerative medicines have the potential to meaningfully shift the efficiency frontier of clinical care, enabling health care systems to accomplish more with the limited resources available, and deliver a higher quality of care while improving outcomes for patients. They could also spur greater investment and economic development, while improving the quality of life for millions of people.

\section{Compliance with Ethics Guidelines}

Conflict of Interest Gil Van Bokkelen serves as Chairman and Chief Executive Officer of Athersys, Inc., which is active in the development of stem cell and regenerative medicine treatments.

Manal Morsy is Vice President and Head of Global Regulatory Affairs at Athersys, Inc., a company with lead interest and activity in the stem cell and regenerative medicine areas.

Toshi-hiko Kobayashi is a senior strategic advisor at Athersys, Inc., (USA), a company with lead interest and activity in the stem cell and regenerative medicine. He is also Chairman of the Alumni Association of Pharmaceutical Sciences at the University of Tokyo (Japan).

Human and Animal Rights and Informed Consent This article does not contain any studies with human or animal subjects performed by any of the authors

\section{References}

Papers of particular interest, published recently, have been highlighted as:

- Of importance

•- Of major importance

1. Sugiura Y, Ju Y-S, Yasuoka J, Jimba M. Rapid increase in Japanese life expectancy after World War II. Biosci Trends. 2010;4(1):9-16.

2. Colombo $\mathrm{F}$ et al. Help wanted? Providing and paying for long term care, OECD Health Policy Studies, OECD Publishing. 2011.

3. Heidenreich PA, Albert NM, Allen LA, Bluemke DA, Butler J, Fonarow GC, et al. Forecasting the impact of heart failure in the United States: a policy statement from the American Heart Association. Circ Heart Fail. 2013;6(3):606-19.

4.• Heidenreich PA, Trogdon JG, Khavjou OA, Butler J, Dracup K, Ezekowitz MD, et al. Forecasting the future of cardiovascular disease in the United States: a policy statement from the American Heart Association. Circulation. 2011;123:(8):933-44. This reference provides an analysis of the projected long term impact of cardiovascular disease. It projects the substantial increase in 
economic impact over the time frame from 2010 to 2030 , driven by key factors such as an aging population.

5. Kowal SL, Dall TM, Chakrabarti R, Storm MV, Jain A. The current and projected economic burden of Parkinson's disease in the United States. Mov Disord. 2013;28(3):311-8.

6. The Ministry of Health Labor and Welfare. National survey of the required number of physicians in hospitals. Japan: Hitsuyou Ishisuu Ni Kannsuru Jittaityousa; 2010. This reference illustrates that the physician shortage is not limited to the USA and Europe.

7. Ishikawa et al. Forecasting the absolute and relative shortage of physicians in Japan using a system dynamics approach. Hum Resour Health. 2013;11:41.

8. European Commission. Commission Staff Working Document on an Action Plan for the EU Health Workforce-SWD. Strasbourg: EC; 2012. This analysis illustrates the current and projected magnitude of the shortage of healthcare workers in the European Union.

9. Nelson AL, Dhimolea E, Reichert JM. Development trends for human monoclonal antibody products. Nat Rev Drug Discov. 2010;9:767-74.

10. Baird LG et al. Accelerated access to innovative medicines for patients in need. Clin Pharmacol Ther. 2014;96(5):559-71.

11. Aggarwal SR. A survey of breakthrough therapy designations. Nat Biotechnol. 2014 32:323-30.

12. Cyranoski D. Japan to offer fast track approval for stem cell therapies. Nat Med. 2013;19(5):510.
13. Azuma K. Regulatory landscape of regenerativemedicine in Japan. Curr Stem Cell Res Ther. 2015. doi:10.1007/s40778-015-0012-6.

14.• Okada K. A new national framework for clinical trials and evaluation of innovative medical care technologies using living cell transplantation in Japan. J Transplant Technol Res. 2014;4(2):137. This reference provides a cogent summary of aspects of the new regulatory framework in Japan that is designed to expedite development of regenerative medicine therapies.

15. Kusakabe T. Regulatory perspectives of Japan. 2014. http://www. pmda.go.jp/regulatory/file/english presentation/biologics/CT-B15kusakabe.pdf. Accessed 12 May 2015.

16. Hara A, Sato D, Sahara Y. New governmental regulatory system for stem cell-based therapies in Japan. Ther Innov Regul Sci. 2014;48(6):681-8.

17. Eichler $\mathrm{H}-\mathrm{G}$ et al. Adaptive licensing: taking the next step in the evolution of drug approval. Clin Pharmacol Ther. 2012;91(3):426-37.

18.• European Medicines Agency. Pilot project on adaptive licensing. 2014. http://www.ema.europa.eu/ema/index.jsp?curl=pages/ news and events/news/2014/03/news detail 002046.jsp\&mid= WC0 01 ac $058004 \mathrm{~d} 5 \mathrm{c} 1$. Accessed 12 May 2015 . This provides additional information and examples of the types of projects that could be considered under the new adaptive licensing framework. 